\title{
NOTE: \\ Dispersibility of Multiwall Carbon Nanotube in a Polyanionic Surfactant Based on UV-Vis Analysis
}

\author{
Yuyun Irmawati ${ }^{1{ }^{* *}}$, Deni Shidqi Khaerudini ${ }^{1}$, Indriyati ${ }^{2}$, Mardiyati ${ }^{3, * *}$, and Rike Yudianti ${ }^{1{ }^{1 * * *}}$ \\ ${ }^{1}$ Research Center for Physics, Indonesian Institute of Sciences, Kawasan Puspiptek Serpong, \\ Tangerang Selatan 15314, Indonesia \\ ${ }^{2}$ Research Unit for Clean Technology, Indonesian Institute of Sciences, \\ Jl. Sangkuriang, Kampus LIPI, Bandung 40135, Indonesia \\ ${ }^{3}$ Department of Materials Science and Engineering, Bandung Institute of Technology, \\ Jl. Ganesha No. 10, Bandung 40132, Indonesia
}

\section{*Corresponding author:}

tel: $+62-21-7560556$

email: yuyun.irmawati@lipi.go.id;

mardiyati@material.itb.ac.id*;

rike.yudianti@lipi.go.id ${ }^{* *}$

Received: May 2, 2019

Accepted: January 8, 2020

DOI: $10.22146 / \mathrm{ijc} .45567$

\begin{abstract}
The degree of carbon nanotube (CNT) dispersion in an ink solution plays a critical role in the performance of CNT based devices. This is a challenging task in the CNT utilization due to strong van der Waals interaction affecting the CNT bundles. A good dispersion degree can be achieved, for instance, by lowering the van der Waals interaction with the strategy of non-covalent interaction between polyanionic surfactant and the CNT surface. Herein, a simple and quick technique to disperse multiwall CNT (MWCNT) by using a polyanionic dispersant, carboxymethyl cellulose (CMC), is reported. The dispersion degree of MWCNT in aqueous solution during the sonication process was studied using UV-Vis analysis. Transmission electron microscope (TEM) was also applied to further investigate the interaction between CMC and MWCNT. The result shows that the maximum dispersion of MWCNT was achieved with a maximum absorbance in the UV-Vis spectra. Higher CMC concentration resulted in a higher viscosity of the solution, thus it increased the sonication duration in obtaining the maximum dispersion. By varying the MWCNT concentration at a constant CMC concentration of $0.25 \mathrm{wt}$.\%, a homogenous MWCNT dispersion was obtained up to $0.2 w t . \%$. The encapsulation of a thin CMC layer on the MWCNT surface with a thickness of 1.5-3 nm was evidenced by TEM micrograph analysis.
\end{abstract}

Keywords: MWCNT; dispersion; carboxymethyl cellulose; UV-Vis spectroscopy

\section{- INTRODUCTION}

Carbon nanotube (CNT), since discovered in 1991 by Iijima [1] has been attracting huge attention due to its unique properties. Owing to high electrical, thermal, mechanical, physical, and chemical properties make CNT to have diverse potential applications such as a transparent conductive electrode, sensing materials, energy storage, adsorbent in water treatment, and filler for conductive nanocomposite [2-6]. The previous study revealed that CNT technology could revolutionize the development of optoelectronic devices. CNT is a promising candidate to substitute indium thin oxide (ITO) that commonly used in display and touch screen applications [7-8]. The ability of CNT to be processed in a solution state (wet method) using simple and fast coating tools such as spraying, spreading, and roll to roll printing also gains its advantages especially for mass production [7]. However, a strong van der Waals interaction between nanotubes that results in the high tendency of CNT to agglomerate is still being a hindrance to its applications. Therefore, producing a good and stable CNT suspension during the fabrication process is a challenging task. 
Various methods have been proposed to decrease the CNT agglomerations (well dispersion). Mechanical dispersions such as ultra-sonication induced dispersion, but it was found to be reversible in short term [9]. Chemical methods based on surface functionalization of CNTs with strong acids such as $\mathrm{HNO}_{3}$ and $\mathrm{H}_{2} \mathrm{SO}_{4}$ create defects on the surface of CNTs. This consequently affects into the CNTs properties [10-11]. Non-covalent methods with surfactants and polymers are more preferable because they have a little influence on the $\pi$-electron cloud of CNTs while preserving the excellent properties of attaching a variety of chemical groups on CNTs surfaces [12]. Amphiphilic polymers or soluble polymers are often used to disperse CNT. The main advantage of using polymers instead of small molecular surfactants is a static dispersion due to multipoint interaction between polymers and the surface of nanotubes to give high dispersion stability [13]. Few studies reported the used of cellulose derivatives for dispersing CNT that yield stable and high CNT concentration in aqueous solution [14-17]. Sodium carboxymethyl cellulose (CMC) has been useful for dispersing single wall carbon nanotube (SWCNT), but only limited information is available about CMC for dispersing the multiwall carbon nanotube (MWCNT).

UV-Vis analysis is beneficial to monitor the debundling dynamics of CNT in suspension in real time and only requires a few volume samples with fast detection time. Consequently, its application can be used to determine the optimal sonication parameters, namely sonication time, surfactant concentration, and the maximum concentration of CNT that probably dispersed evenly in the suspension. Therefore, in this study, a fast and simple method to analyze the degree of MWCNT dispersion with CMC as a surfactant was performed by using UV-Vis spectroscopy. In addition, TEM analyses were conducted to analyze the dispersion mechanism and to investigate the interaction between MWCNT and CMC.

\section{- EXPERIMENTAL SECTION}

\section{Materials}

The commercially pristine MWCNT (purity of 95\%, the outer diameter range of $20-50 \mathrm{~nm}$, and the length of around $5 \mu \mathrm{m}$ ) was purchased from Heji Inc., Hongkong.
A high viscosity carboxymethyl cellulose (CMC) from Sigma Aldrich, USA was used. Both materials were used as received. Deionized (DI) water from water purification (TKA-pacific, Thermofisher scientific) was used for all sample preparation.

\section{Instrumentation}

The sonication process of MWCNT-CMC suspension was performed using a $750 \mathrm{~W}$ ultrasonic horn (Sonic Vibracell-750W). The absorbance spectra of MWCNT colloids were measured by a UV-Vis spectrophotometer (Carry 60, Agilent Technologies). The interaction between MWCNT and CMC was studied using images of a high-resolution TEM (Tecnai G2 STWIN).

\section{Procedure}

MWCNT suspensions were prepared using the following method. CMC $(0.25,0.5$, and 1 wt.\%) was dissolved in $50 \mathrm{~mL}$ DI water. Pristine MWCNT (0.1 wt.\%) was added into those three diffident $\mathrm{CMC}$ concentration solutions and stirred for $30 \mathrm{~min}$. The dissolution was performed with the ultra-sonication process using an ultrasonic horn that was set to the amplitude of $20 \%$ with pulse mode ( $6 \mathrm{sec}$ on and $2 \mathrm{sec}$ off). In order to protect the mixture from being over-heated, the beaker was positioned in a medium of ice-cooled water. Sonication time was varied from 15 to $75 \mathrm{~min}$. The degree of MWCNT dispersion was studied by measuring the absorbance spectra using a UV-Vis spectrophotometer at 200-800 nm in a quartz cuvette with a path length of $10 \mathrm{~mm}$. For optimizing the sonication time, samples were taken regularly along the sonication process. All samples were diluted several times in which samples absorbance was suitable for UV-Vis analysis. CMC aqueous solution with the same concentration as in the MWCNT samples was set as a reference to subtract the absorbance spectra of CMC in the suspension. Finally, the MWCNT coated CMC was confirmed by using the TEM micrograph. Samples for TEM analysis were prepare by diluting the dispersed MWCNT until the samples were suitable for TEM analysis. Furthermore, TEM samples were dropped onto $\mathrm{Cu}$-grid carbon layer and then dried in air. TEM imaging process was operated at accelerate voltage of $200 \mathrm{kV}$. 
To estimate the maximum dispersibility, MWCNT concentrations in suspension were varied at $0.1,0.2,0.3$, and 0.4 wt.\% with a constant CMC concentration at 0.25 wt.\% designated as MWCNT-01, MWCNT-02, MWCNT-03, and MWCNT-04, respectively. Dispersion time was set at $60 \mathrm{~min}$. All different concentrations of MWCNT suspension were centrifuged at $8000 \mathrm{rpm}$ for $20 \mathrm{~min}$ for precipitation test purposes. The supernatant (about $70 \%$ of the upper level of suspension) from four various MWCNT concentrations was diluted several times to measure their UV-Vis absorbance and the real MWCNT concentration was then calculated by using standard UV-Vis curve.

\section{- RESULTS AND DISCUSSION}

Fig. 1(a) shows the absorbance spectra of MWCNT (0.1 wt.\%) suspension with a CMC concentration of $0.25 \mathrm{wt} . \%$ as a function of sonication time. It is noticeable that the absorbance peaks increase with respect to sonication time. Generally, the spectra reveal the highest peak mainly between $200-300 \mathrm{~nm}$ and then decrease gradually to $800 \mathrm{~nm}$. In this result, the highest absorbance peak is located at about $266 \mathrm{~nm}$ which is similar and comparable with the previous results from [18-19]. The absorbance peak in MWCNT is associated with the $\pi-\pi^{*}$ transition in the electronic band that will also appear for all $\mathrm{sp}^{2}$ carbon allotropes [20]. The absorbance peak is strongly associated with MWCNT dispersion. MWCNT is inactive in UV-Vis spectra when in bundles (agglomerate) state due to some tunneling effects of the tubes to give no absorbance peaks. Therefore, gaining area
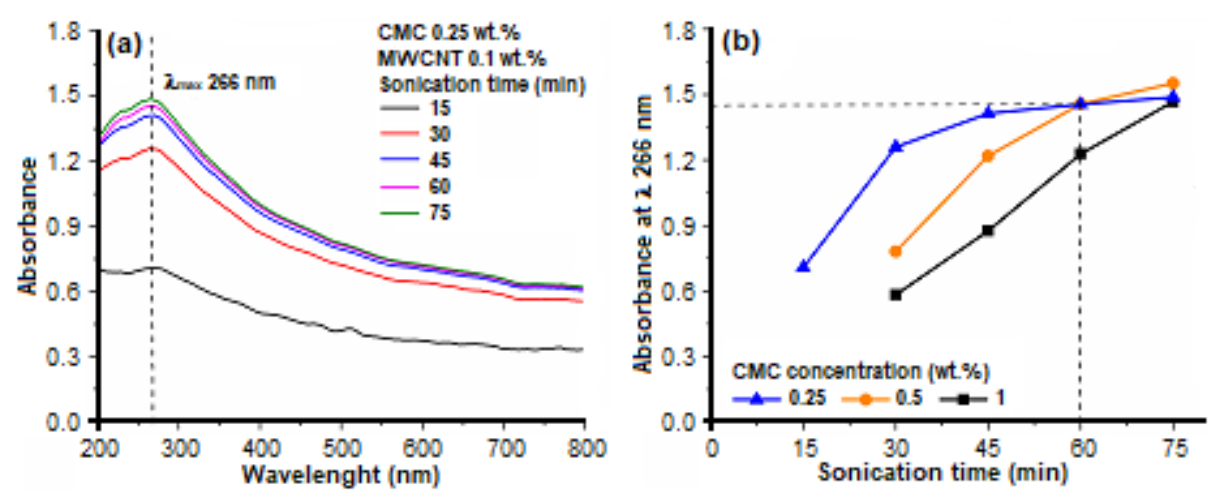

Fig 1. (a) UV-Vis spectra of MWCNT suspension with CMC concentration of $0.25 \mathrm{wt} . \%$ and (b) the absorbance peaks at $266 \mathrm{~nm}$ as a function of sonication time for different CMC concentration

Yuyun Irmawati et al. below the peak spectra means an increase in the amount of exfoliated MWCNT. From Fig. 1(a), there is a significant increase in the absorbance spectra after $15 \mathrm{~min}$. This can be explained due to the massive exfoliation of a big part of MWCNT bundles in the initial stage. In the next stages, there is such an extend exfoliation from a small part of MWCNT bundles into MWCNT single tubes. Therefore, only a gradual increase in the absorbance spectra can be observed. In this work, the sonication time was set at a maximum of 75 min, since a longer sonication time could affect a tube fragmentation and destruction from their high local shear of ultrasonic wave [10].

The results of the effect of CMC concentration (0.25, 0.5, and 1 wt.\%) at constant MWCNT loading (0.1 wt.\%) are shown in Fig. 1(b). In general, the absorbance peak for all conditions increases along with sonication time. Among all conditions, the absorbance number for CMC $0.25 \mathrm{wt} . \%$ is relatively higher than the two other CMC condition. At the sonication time of $15 \mathrm{~min}$, only CMC $0.25 \mathrm{wt} . \%$ shows absorption at $266 \mathrm{~nm}$. Then, the spectra increase significantly for $30 \mathrm{~min}$ (from 0.7 to 1.3 ) and slightly increase for $45 \mathrm{~min}$. Then, the absorbance peak remains constant at 1.5 for $60 \mathrm{~min}$. This indicates that the sonication degree of the MWCNT has reached saturation. Meanwhile, for CMC 0.5 and 1 wt.\%, they still show some upward trend. Fig. 1(b) also reveals that higher $\mathrm{CMC}$ concentration implies a higher viscosity of the suspension that requires longer sonication time to reach their saturation. This can be explained due to a higher suspension viscosity that makes harder for the 
penetration of ultrasonic waves and minimizes their Brownian movement of the nanotube during the exfoliation. The previous study showed that nanotube dispersion was more effective in the solution at low viscosity [11]. Thus, in this study, the CMC 0.25 wt.\% is the optimum result due to its shortest saturation time. This short sonication time is preferable since the longer one could reduce the aspect ratio of the tube. It will also decrease their mechanical, electrical, and thermal properties of the tube [10].

During the dispersion process, a polyanionic surfactant (CMC) was absorbed onto the nanotube surface. Fig. 2 shows TEM image analyses indicating a thin layer of CMC covering the surface of MWCNT nanotubes. Before sonication, the pristine MWCNT consists of many tube bundles (Fig. 2(a)) and an individual tube of uncoated surfaces (Fig. 2(b)). After sonication, the bundles are encapsulated with the CMC layer as shown in Fig. 2(c) and 2(d) with a thickness of about $1.5-3 \mathrm{~nm}$. Zhao et al. also observed that the CMC thin layer on the MWCNT surface is in the range of 5.4 $\mathrm{nm}$ [21]. Those mechanisms are agreed upon and can be explained as proposed by Strano et al. [22]. Fig. 3 illustrates the dispersion mechanism during the sonication process in the presence of CMC surfactant. The dispersion process by applying a high frequency of an ultrasonic wave is started by the formation of inter- bundles end-gaps caused by a high local share of the ultrasonic wave. This process is called the "unzippering" mechanism [22-23]. These gaps act as absorption sites for the CMC. Due to the presence of CMC, the gap distance between tubes becomes larger meaning that van der Waal's interaction is minimized. Then, the debundling process continually occurs until all MWCNT bundles exfoliated or reach their saturation state. Therefore, the degree of exfoliating is directly associated with the increase in absorption spectra.

\section{Precipitation Test of MWCNT Dispersion}

In order to measure the dispersion stability, MWCNT suspensions with four different concentrations (0.1-0.4 wt.\%) at a constant CMC concentration of 0.25 wt.\% were precipitated by centrifugation. Fig. 4(a) shows the UV-Vis spectra for all MWCNT suspension after normalization (samples have been diluted previously by a certain factor to having a similar concentration). The normalization was carried out in order to gain a similar response to absorbance. However, as shown in the insert Fig. 4(a), the spectra peak of MWCNT-02 at $266 \mathrm{~nm}$ is 0.8 ; and then decrease significantly to 0.7 for MWCNT04 . This is assumed that at high MWCNT concentration, the amount of CMC is not sufficient to encapsulate the nanotube surface. In other words, only a small amount of MWCNT surface that can be covered by CMC to
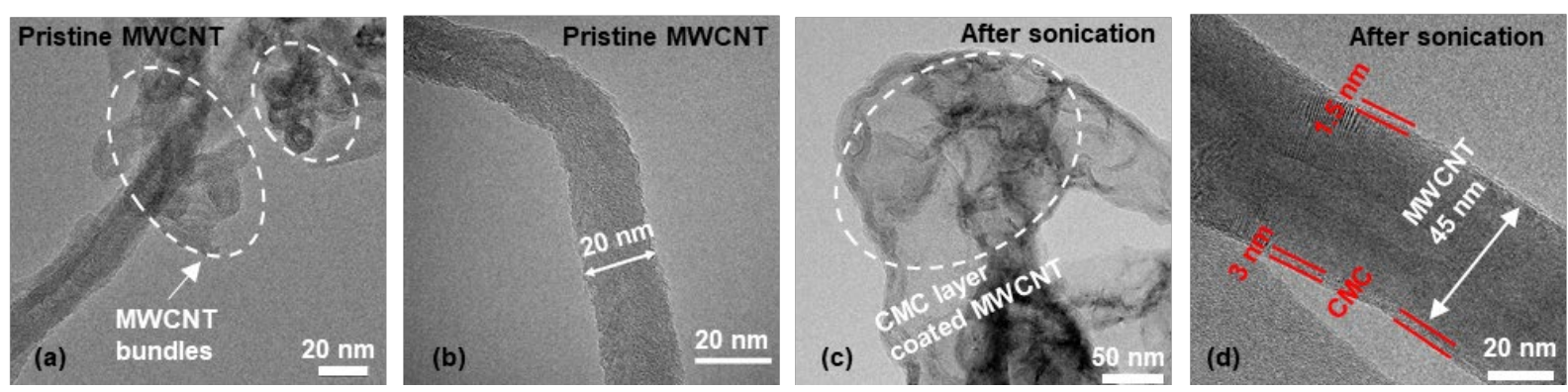

Fig 2. TEM images of pristine MWCNT (a-b) before and (c-d) after sonication

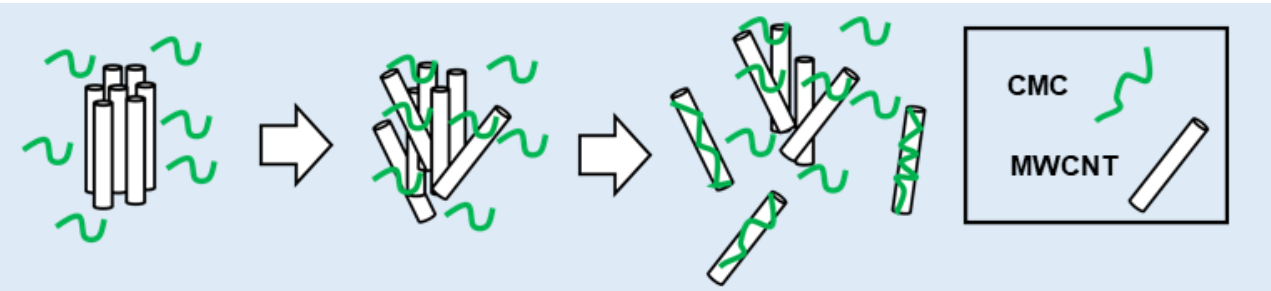

Fig 3. Mechanism of MWCNT exfoliation from bundles during the sonication process using CMC 

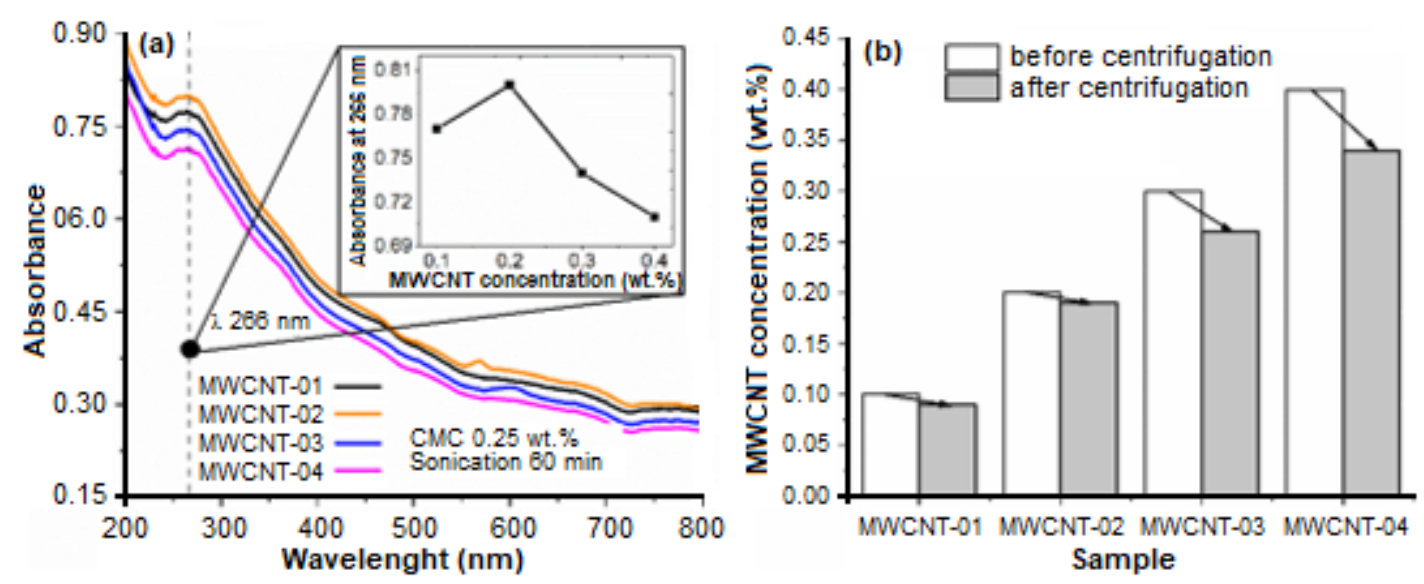

Fig 4. (a) UV-Vis spectra of the suspension as a function of MWCNT concentration and (b) MWCNT concentration before and after centrifugation at suspension using CMC 0.25 wt.\%

minimize van der Waals interaction. Therefore, only a certain amount of MWCNT can be dispersed properly. From the previous study, CMC was able to disperse SWCNT effectively, however it was only up to a concentration of $0.12 \mathrm{wt} . \%$ [24].

Furthermore, Fig. 4(b) presents the change in the MWCNT concentration before and after centrifugation that can be used to calculate the precipitation degree. The amount of MWCNT precipitate increases for higher MWCNT concentration since it cannot maintain the colloidal stability during centrifugation. In this work, the surfactant (CMC) was set only at $0.25 \mathrm{wt} . \%$. Therefore, more MWCNT concentration results in a higher precipitation degree. In the previous study, the inverse of precipitation degree, which is the ratio of MWCNT concentration after and before centrifugation, was used to calculate the degree of extractability that determines the ability of surfactant to disperse MWCNT in a certain ratio [12]. In this study, the maximum degree of extractability, the ratio of MWCNT concentration at the initial stage compared to after centrifugation process, is achieved about 0.95 for MWCNT-02. This value is slightly higher than that the degree of extractability of commercially available surfactants, i.e., Triton X-100TM, sodium dodecyl sulfate (SDS), and Tween-80TM which are 0.90, 0.84 , and 0.89 , respectively [12]. Moreover, from the previous studies, the nanotube suspension that used CMC remained stable for over one year [24], while for the SDS, the suspension could stand only for $500 \mathrm{~h} \mathrm{[25].}$

\section{- CONCLUSION}

It was noticeable that $\mathrm{UV}$-Vis analysis is a powerful method to investigate the dispersion degree of MWCNT as directly associated with the absorbance spectra. A well dispersed MWCNT suspension was obtained by the sonication process using the CMC surfactant. The encapsulation of CMC layer on the surface of MWCNT indicates that the van der Waals interaction is successfully minimized. Furthermore, by varying the MWCNT concentration and precipitation test, it showed that CMC has a good dispersibility and high extractability.

\section{- ACKNOWLEDGMENTS}

This work was supported by Kemenristekdikti under Master Degree Scholarship Program (2015). The authors acknowledge research facilities support from RC for Physics LIPI and Research Unit for Clean Technology LIPI. Yuyun acknowledges to Dr. Rakhmat Hidayat (Physics Dept. ITB) for the valuable discussion.

\section{- REFERENCES}

[1] Iijima, S., 1991, Helical microtubles of graphitic carbon, Nature, 354, 56-58.

[2] Shah, K.A., and Tali, B.A., 2016, Synthesis of carbon nanotubes by catalytic chemical vapour deposition: A review on carbon sources, catalysts and substrates, Mater. Sci. Semicond. Process., 41, 67-82.

[3] Zhou, G., Byun, J.H., Oh, Y., Jung, B.M., Cha, H.J., 
Seong, D.G., Um, M.K., Hyun, S., and Chou, T.W., 2017, Highly sensitive wearable textile-based humidity sensor made of high-strength, single-walled carbon nanotube/poly(vinyl alcohol) filaments, ACS Appl. Mater. Interfaces, 9 (5), 4788-4797.

[4] Wang, F., Kozawa, D., Miyauchi, Y., Hiraoka, K., Mouri, S., Ohno, Y., and Matsuda, K., 2015, Considerably improved photovoltaic performance of carbon nanotube-based solar cells using metal oxide layers, Nat. Commun., 6, 6305.

[5] Mubarak, N.M., Sahu, J.N., Abdullah, E.C., and Jayakumar, N.S., 2016, Rapid adsorption of toxic $\mathrm{Pb}$ (II) ions from aqueous solution using multiwall carbon nanotubes synthesized by microwave chemical vapor deposition technique, J. Environ. Sci., 45, 143-155.

[6] Yan, J., and Jeong, Y.G., 2015, Highly elastic and transparent multiwalled carbon nanotube/poly dimethylsiloxane bilayer films as electric heating materials, Mater. Des., 86, 72-79.

[7] Zhou, Y., and Azumi, R., 2016, Carbon nanotube based transparent conductive films: Progress, challenges, and perspectives, Sci. Technol. Adv. Mater., 17 (1), 493-516.

[8] Yu, L.P., Shearer, C., and Shapter, J., 2016, Recent development of carbon nanotube transparent conductive films, Chem. Rev., 116 (22), 13413-13453.

[9] Konsta-Gdoutos, M.S., Metaxa, Z.S., and Shah, S.P., 2010, Highly dispersed carbon nanotube reinforced cement based materials, Cem. Concr. Res., 40 (7), 1052-1059.

[10] Dassios, K.G., Alafogianni, P., Antiohos, S.K., Leptokaridis, C., Barkoula, N.M., and Matikas, T.E., 2015, Optimization of sonication parameters for homogeneous surfactant-assisted dispersion of multiwalled carbon nanotubes in aqueous solutions, J. Phys. Chem. C, 119 (13), 7506-7516.

[11] Ma, P.C., Siddiqui, N.A., Marom, G., and Kim, J.K., 2010, Dispersion and functionalization of carbon nanotubes for polymer-based nanocomposites: A review, Composites Part A, 41 (10), 1345-1367.

[12] Rastogi, R., Kaushal, R., Tripathi, S.K., Sharma, A.L., Kaur, I., and Bharadwaj, L.M., 2008, Comparative study of carbon nanotube dispersion using surfactans, J. Colloid Interface Sci., 328 (2), 421-428.

[13] Fujigaya, T., and Nakashima, N., 2015, Noncovalent polymer wrapping of carbon nanotubes and the role of wrapped polymers as functional dispersants, Sci. Technol. Adv. Mater., 16 (2), 024802.

[14] Imazu, N., Fujigaya, T., and Nakashima, N., 2014, Fabrication of flexible transparent conductive films from long double-walled carbon nanotubes, Sci. Technol. Adv. Mater., 15 (2), 025005.

[15] Wang, Y., Yang, H.J., Geng, H.Z., Zhang, Z.C., Ding, E.X., Meng, Y., Luo, Z.J., Wang, J., Su, X.M., and Da, S.X., 2015, Fabrication and evaluation of adhesion enhanced flexible carbon nanotube transparent conducting films, J. Mater. Chem. C, 3 (15), 3796-3802.

[16] Hamedi, M.M., Hajian, A., Fall, A.B., Håkansson, K., Salajkova, M., Lundell, F., Wågberg, L., and Berglund, L.A., 2014, Highly conducting, strong nanocomposites based on nanocellulose-assisted aqueous dispersions of single-wall carbon nanotubes, ACS Nano, 8 (3), 2467-2476.

[17] Dadfar, S.M.M., and Kavoosi, G., 2014, Mechanical and water binding properties of carboxymethyl cellulose/multiwalled carbon nanotube nano composites, Polym. Compos., 36 (1), 145-152.

[18] Yu, J., Grossiord, N., Koning, C.E., and Loos, J., 2007, Controlling the dispersion of multi-wall carbon nanotubes in aqueous surfactant solution, Carbon, 45 (3), 618-623.

[19] Shi, Y., Ren, L., Li, D., Gao, H., and Yang, B., 2013, Optimization conditions for single-walled carbon nanotubes dispersion, J. Surf. Eng. Mater. Adv. Technol., 3, 6-12.

[20] Salvatierra, R.V., Cava, C.E., Roman, L.S., and Zarbin, A.J.G., 2013, ITO-free and flexible organic photovoltaic device based on high transparent and conductive polyaniline/carbon nanotube thin films, Adv. Funct. Mater., 23 (12), 1490-1499.

[21] Zhao, T.K., Liu, L.H., Li, G.M., Du, L., Zhao, X., Yan, J., Cheng, Y.L., Dang, A.L., and Li, T.H., 2012, Preparation and electrochemical property of 
CMC/MWCNT composite using ionic liquid as the solvent, Chin. Sci. Bull., 57, 1620-1625.

[22] Strano, M.S., Moore, V.C., Miller, M.K., Allen, M.J., Haroz, E.H., Kittrell, C., Hauge, R.H., and Smalley, R.E., 2003, The role of surfactant adsorption during ultrasonication in the dispersion of single-walled carbon nanotubes, J. Nanosci. Nanotechnol., 3 (1-2), 81-86.

[23] Bandyopadhyaya, R., Nativ-Roth, E., Regev, O., and Yerushalmi-Rozen, R., 2002, Stabilization of individual carbon nanotubes in aqueous solutions, Nano Lett., 2 (1), 25-28.

[24] Minami, N., Kim, Y., Miyashita, K., Kazaoui, S., and Nalini, B., 2006, Cellulose derivatives as excellent dispersants for single-wall carbon nanotubes as demonstrated by absorption and photoluminescence spectroscopy, Appl. Phys. Lett., 88, 093123.

[25] Jiang, L., Gao, L., and Sun, J., 2003, Production of aqueous colloidal dispersions of carbon nanotubes, J. Colloid Interface Sci., 260 (1), 89-94. 